\title{
Experimental investigation of Delay Time Problem in Galactic Cosmic Rays Stream in Solar Cycles: from 19 to 24
}

\author{
1.Marek Siluszyk 1 \\ Siedlce University \\ 3-maja 54, 08-110 Siedlce, Poland \\ E-mail:marek.siluszyk@uph.edu.pI
}

\section{Krzysztof Iskra}

\author{
Polish Air Force \\ UniversityDeblin, Poland \\ E-mail:k.iskrallaw.mil.pI

\section{Witold} \\ Wozniak \\ Polish Gas \\ CompanyWars \\ aw, Poland \\ E-mail:witek.wozniak81@gmail.com
}

\section{Michael Alania}

\author{
Institute of Geophysics, Tbilisi State \\ UniversityTbilisi, Georgia \\ E-mail:alanialuph.edu.pI
}

We investigate long period changes of Galactic Cosmic Rays (GCR) in different epochs of Solar Magnetic Cycles(SMC). The purpose of this paper is to carry on a study of the Delay Time (DT) between the changes of GCR intensity on the one hand and various parameters characterizing conditions in heliosphere, on the other.We prove the existence of varying DT between the changes of GCR intensity and parameters characterizing Solar Activity (SA), as the SunSpot Number (SSN) and Tilt Tngle(TA).Based on the experimental investigation we obtained different DT's for various sign of the Global Solar Magnetic Field (GSMF) in the considered period 1959-2014. We have showed that the DT for periods with a negative sign $(A<0)$ of the GSMF is greater than for periods $(A>0)$ with a positive sign. The main reason for this phenomenon is drift streams of GCR caused by the gradient and curvature of the Interplanetary Magnetic Field (IMF). The study of the DT invarious cycles of SA and depending on the sign of the GSMF is very important from the point of view of the theoretical modeling of the propagation of GCR particles in the heliosphere. 